\title{
Axisymmetric Quadrilateral Elements for Large Deformation Hyperelastic Analysis
}

\author{
G.H.Liu, K.Y.Sze* \\ Department of Mechanical Engineering, The University of Hong Kong, \\ Pokfulam Road, Hong Kong SAR, P.R.CHINA \\ *Email: kysze@hku.hk
}

\begin{abstract}
In this paper, axisymmetric 8-node and 9-node quadrilateral elements for large deformation hyperelastic analysis are devised. To alleviate the volumetric locking which may be encountered in nearly incompressible materials, a volumetric enhanced assumed strain (EAS) mode is incorporated in the eight-node and nine-node uniformly reduced-integrated (URI) elements. To control the compatible spurious zero energy mode in the 9-node element, a stabilization matrix is attained by using a hybridstrain formulation and, after some simplification, the matrix can be programmed in the element subroutine without resorting to numerical integration. Numerical examples show the relative efficacy of the proposed elements and other popular eight-node elements. In view of the constraint index count, the two elements are analogous to the Q8/3P and Q9/3P elements based on the u-p hybrid/mixed formulation. However, the former elements are more straight forward than the latter elements in both formulation and programming implementation.
\end{abstract}

Keywords: hybrid/mixed, finite element method, large deformation, hyperelastic, volumetric locking, enhanced assumed strain, stabilization

Published in International Journal of Mechanics and Materials in Design, 2010, v. 6(3) p. 197-207 


\section{INTRODUCTION}

Hyperelastic constitutive models are commonly used for rubbery materials and biological soft tissues which are often assumed to be nearly incompressible. In finite element analysis of nearly incompressible materials, when the number of volumetric constraints per element is excessive, the element assemblage would exhibit the volumetric or dilatational locking which is signified by a nonphysical and excessive stiffness as if the assemblage was locked. Meanwhile, the predicted pressure or mean stress may exhibit oscillation [1-11]. In four-node quadrilateral elements, the latter phenomenon is known as the checkerboard mode [4,5]. Note worthily, an effective way of tackling nearly incompressible material analyses is the u-p formulation in which a variational functional with independently assumed displacement and pressure is employed $[2,9,10]$.

Over the past decades, effort has been put into the development of high performance finite element formulations for axisymmetric problems. While incompatible plane and solid QM6 elements [12] have been popularly employed by commercial software, the related axisymmetric element fails the patch test. On the other hand, a number of incompatible and enhanced assumed strain (EAS) axisymmetric elements which manage to patch test were derived [13-16] years later. Based on multifield functionals and their variants, there have been quite a number of hybrid/mixed axisymmetric elements derived [5,14-21]. Stabilized axisymmetric element can also be noted [22]. Apart from the well-known advanced finite element formulations, two approaches to reduce the number of dilatational constraints in axisymmetric elements were proposed by Yu \& his coworkers [7,8,11]. In the first approach, $r u_{r}$ (the product of the radial coordinate $r$ and radial displacement $u_{r}$ ) is employed instead of $u_{r}$ as the nodal dofs. In the second approach, $r^{2}$ and instead of $r$ are interpolated in the element formulation.

Note worthily, many of the afore-reviewed elements are restricted to linear analyses. In the present study, an eight-node quadrilateral element (Q8) and a nine-node quadrilateral element (Q9) are devised for large deformation axisymmetric analyses of hyperelastic materials. Using the uniformly reduced integration (URI), volumetric locking in the Q8 and Q9 elements is alleviated by adopting a volumetric EAS mode which is selected with respect to the principles of EAS method. The practice is similar to the destabilization approach $[9,23]$ used to reduce the rank of the penalty matrix. The enhanced URI Q8 element possesses only one incompatible spurious zero energy mode which is always suppressed if there are more than one element in the mesh. The enhanced URI Q9 element, however, possesses two compatible spurious zero energy modes. To suppress them, stabilization matrix is derived by a hybridstrain method [23]. A notable feature of the stabilization matrix is that it can be derived without using numerical integration. 
A number of numerical examples are considered. In most of them, the proposed elements and Abaqus's URI Q8 and hybrid URI Q8 (with three independently assumed pressure modes) [24] yield close predictions. However, when the problem domains are highly restrained and the material is nearly incompressible, pressure predictions of the enhanced URI Q8 and Abaqus's hybrid URI Q8 are oscillatory whereas the predictions of the enhanced URI Q9 element remains smooth.

\section{DISPLACEMENT-DERIVED AND ENHANCED ASSUMED STRAINS}

Figure 1 shows the Q9 element on the $r$-z-plane. In the absence of the centre node, it becomes the Q8 element. Within the element, the initial coordinate vector $\mathbf{x}$ and the displacement vector $\mathbf{u}$ are interpolated, i.e.

$$
\mathbf{x}(\xi, \eta)=\left\{\begin{array}{l}
r(\xi, \eta) \\
z(\xi, \eta)
\end{array}\right\}=\sum_{j=1}^{n} N_{j}(\xi, \eta) \mathbf{x}_{j}=\mathbf{N}(\xi, \eta) \underline{\mathbf{x}} \quad, \quad \mathbf{u}(\xi, \eta)=\left\{\begin{array}{l}
u_{r}(\xi, \eta) \\
u_{z}(\xi, \eta)
\end{array}\right\}=\sum_{j=1}^{n} N_{j}(\xi, \eta) \mathbf{u}_{j}=\mathbf{N}(\xi, \eta) \underline{\mathbf{u}}
$$

where $(\xi, \eta)$ are the natural coordinates; $\mathbf{x}_{j} \mathrm{~s}$ and $\mathbf{u}_{j} \mathrm{~s}$ are the nodal counterparts of $\mathbf{x}$ and $\mathbf{u}$, respectively; $n$ equals 8 and 9 for the Q8 and Q9 elements, respectively. The interpolation functions $N_{j} \mathrm{~s}$, which are different for Q8 and Q9 elements, can be found in standard textbooks and are not repeated here. Moreover,

$$
\mathbf{N}(\xi, \eta)=\left[N_{1} \mathbf{I}_{2}, \ldots, N_{n} \mathbf{I}_{2}\right], \underline{\mathbf{x}}=\left\{\begin{array}{c}
\mathbf{x}_{1} \\
\vdots \\
\mathbf{x}_{n}
\end{array}\right\}, \underline{\mathbf{u}}=\left\{\begin{array}{c}
\mathbf{u}_{1} \\
\vdots \\
\mathbf{u}_{n}
\end{array}\right\}
$$

in which $\mathbf{I}_{m}$ is the $m$-th order identity matrix. The natural Green strain component $e_{i j}$ 's are:

$$
e_{i j}=\left(\mathbf{x},{ }_{i}^{T} \mathbf{u},{ }_{j}+\mathbf{x},{ }_{j}^{T} \mathbf{u}_{i}\right) / 2+\left(\mathbf{u},{ }_{i}^{T} \mathbf{u},{ }_{j}+\mathbf{u}_{j}^{T} \mathbf{u}_{i}\right) / 4 \quad \text { for } \quad i, j=\xi, \eta
$$

and the vector of physical Green strain components can be obtained as:

$$
\boldsymbol{\varepsilon}=\left\{\varepsilon_{\theta \theta}, \varepsilon_{r r}, \varepsilon_{z z}, 2 \varepsilon_{r z}\right\}^{T}=\mathbf{T}\left\{\varepsilon_{\theta \theta}, \varepsilon_{\xi \xi}, \varepsilon_{\eta \eta}, 2 \varepsilon_{\xi \eta}\right\}^{T}
$$

in which 


$$
\varepsilon_{\theta \theta}=\frac{u_{r}}{r}+\frac{1}{2}\left(\frac{u_{r}}{r}\right)^{2} \text { and } \mathbf{T}=\left[\begin{array}{cccc}
1 & 0 & 0 & 0 \\
0 & \frac{\partial r}{\partial \xi} \frac{\partial r}{\partial \xi} & \frac{\partial z}{\partial \xi} \frac{\partial z}{\partial \xi} & \frac{\partial r}{\partial \xi} \frac{\partial z}{\partial \xi} \\
0 & \frac{\partial r}{\partial \eta} \frac{\partial r}{\partial \eta} & \frac{\partial z}{\partial \eta} \frac{\partial z}{\partial \eta} & \frac{\partial r}{\partial \eta} \frac{\partial z}{\partial \eta} \\
0 & 2 \frac{\partial r}{\partial \xi} \frac{\partial r}{\partial \eta} & 2 \frac{\partial z}{\partial \xi} \frac{\partial z}{\partial \eta} & \frac{\partial r}{\partial \xi} \frac{\partial z}{\partial \eta}+\frac{\partial z}{\partial \xi} \frac{\partial r}{\partial \eta}
\end{array}\right]^{-1}
$$

Among the first and second order plane and axisymmetric u-p formulated elements [2,9,10], the most robust one is properly the Q9-3P element which is the Q9 element with three assumed pressure modes $[9,10]$. Naturally, the three pressure modes can be taken to be $1, \xi$ and $\eta$.

For the URI Q8 and Q9 element, the integration rule is the $2 \times 2$ quadrature. As the strain and thus the pressure are sampled at four integration points, their characteristics in regard to nearly incompressible materials should be similar to the u-p formulated elements with four pressure modes which can be taken to be $1, \xi, \eta$ and $\xi \eta$. To nullify the effect of the fourth pressure mode without explicitly defining a pressure field, one may incorporate a volumetric EAS mode $\xi \eta$. This reduces the rank of the penalty matrix formed by $2 \times 2$ quadrature by one and the similar technique has been employed in references [9,23]. It is expected that enhanced (with the EAS field) Q8 URI elements yield predictions similar to the element with three pressure modes explicitly assumed such as ABAQUS's CAX8RH element [24].

As the integral of the EAS field over the element domain must be zero for patch test fulfillment [13] and the integral over the un-deformed element domain $\Omega^{e}$ can be manipulated as:

$$
\int_{\Omega^{e}} \circ d \Omega=\langle\circ\rangle=2 \pi \int_{-1-1}^{+1+1} \int_{-1}^{+1} \circ J d \xi d \eta
$$

in which $J=(\partial r / \partial \xi)(\partial z / \partial \eta)-(\partial r / \partial \eta)(\partial z / \partial \xi)$ is the Jacobian determinant, the volumetric EAS mode $\xi \eta$ can be modified to be

$$
\boldsymbol{\varepsilon}^{E A S}=\frac{\xi \eta}{r J}[1,1,1,0]^{T} \alpha=\mathbf{E} \alpha
$$

where $\alpha$ is the scalar coefficient, the shape function matrix $\mathbf{E}$ is given by the underlined terms. The union of (4) and (5) leads to the enhanced strain:

$$
\boldsymbol{\varepsilon}=\left[\boldsymbol{B}^{\mathrm{x}+\mathbf{u} / 2}, \boldsymbol{E}\right]\left\{\begin{array}{l}
\underline{\mathbf{u}} \\
\alpha
\end{array}\right\} \text { where }
$$

where 


$$
\mathbf{B}^{\mathbf{x}}=\mathbf{T}\left[\begin{array}{c}
\frac{1}{r^{2}} \underline{\mathbf{x}}^{T} \mathbf{N}^{T}\left[\begin{array}{ll}
1 & 0 \\
0 & 0
\end{array}\right] \boldsymbol{N} \\
\underline{\mathbf{x}}^{T} \mathbf{N}_{\xi}^{T} \mathbf{N},{ }_{\xi} \\
\underline{\mathbf{x}}^{T} \mathbf{N}_{\eta}^{T} \mathbf{N},{ }_{\eta} \\
\underline{\mathbf{x}}^{T}\left(\mathbf{N},{ }_{\xi}^{T} \mathbf{N},{ }_{\eta}+\mathbf{N},{ }_{\eta}^{T} \mathbf{N},{ }_{\xi}\right)
\end{array}\right] .
$$

\section{THE ENHANCED URI ELEMENTS}

For the intended large deformation hyperelastic analysis, let $(\mathbf{u}, \alpha)$ and $(\mathbf{u}+\Delta \mathbf{u}, \alpha+\Delta \alpha)$ be respectively the acquired and refined approximate solutions to the nonlinear equilibrium problem. The virtual work principle for an assemblage of elements $e$ 's can be expressed as:

$$
\sum_{e} \delta U^{e}=\delta W
$$

where $\delta$ is the virtual symbol and $\delta W$ is the external virtual work. Moreover,

$$
\delta U^{e}=\left\langle(\boldsymbol{\sigma}+\mathbf{C} \Delta \boldsymbol{\varepsilon})^{T} \delta \Delta \boldsymbol{\varepsilon}\right\rangle
$$

is the elemental internal virtual work. For hyperelastic materials, the vector of second Piola-Kirchhoff stress components $\sigma$ and tangential material stiffness matrix $\mathbf{C}$ can be derived from the strain energy function $A(\boldsymbol{\varepsilon})$ as [24] :

$$
\boldsymbol{\sigma}=\frac{\partial A(\boldsymbol{\varepsilon})}{\partial \boldsymbol{\varepsilon}}, \mathbf{C}=\frac{\partial}{\partial \boldsymbol{\varepsilon}}\left(\frac{\partial A(\boldsymbol{\varepsilon})}{\partial \boldsymbol{\varepsilon}}\right)^{T}
$$

Further from (6) and (13),

$$
\Delta \boldsymbol{\varepsilon}=\left.\boldsymbol{\varepsilon}\right|_{(\underline{\mathbf{u}}+\Delta \underline{\mathbf{u}}, \alpha+\Delta \alpha)}-\left.\boldsymbol{\varepsilon}\right|_{(\underline{\mathbf{u}}, \alpha)}=\left[\mathbf{B}^{\mathbf{x}+\mathbf{u}+\Delta \mathbf{u} / 2}, \mathbf{E}\right]\left\{\begin{array}{c}
\Delta \underline{\mathbf{u}} \\
\Delta \alpha
\end{array}\right\}, \delta \Delta \boldsymbol{\varepsilon}=\left[\mathbf{B}^{\mathbf{x}+\mathbf{u}+\Delta \underline{u}, \mathbf{E}]}\left\{\begin{array}{l}
\delta \Delta \underline{\mathbf{u}} \\
\delta \Delta \alpha
\end{array}\right\}\right.
$$

Thus,

$$
\delta U^{e}=\left\{\begin{array}{c}
\delta \Delta \underline{\mathbf{u}} \\
\delta \Delta \alpha
\end{array}\right\}^{T}\left\langle\left[\mathbf{B}^{\mathbf{x}+\mathbf{u}+\Delta \mathbf{u}}, \mathbf{E}\right]^{T}\left(\boldsymbol{\sigma}+\mathbf{C}\left[\mathbf{B}^{\mathbf{x}+\mathbf{u}+\Delta \mathbf{u} / 2}, \mathbf{E}\right]\left\{\begin{array}{c}
\Delta \underline{\mathbf{u}} \\
\Delta \alpha
\end{array}\right\}\right)\right\rangle
$$

By linearizing with respect to the incremental terms,

$$
\delta U^{e}=\left\{\begin{array}{c}
\delta \Delta \underline{\mathbf{u}} \\
\delta \Delta \alpha
\end{array}\right\}^{T}\left(\left\langle\left[\mathbf{B}^{\mathbf{x}+\mathbf{u}}+\mathbf{B}^{\Delta \mathbf{u}}, \mathbf{E}\right]^{T} \boldsymbol{\sigma}\right\rangle+\left\langle\left[\mathbf{B}^{\mathbf{x}+\mathbf{u}}, \mathbf{E}\right]^{T} \mathbf{C}\left[\mathbf{B}^{\mathbf{x}+\mathbf{u}}, \mathbf{E}\right]\right\rangle\right)\left\{\begin{array}{c}
\Delta \underline{\mathbf{u}} \\
\Delta \alpha
\end{array}\right\}
$$




$$
=\left\{\begin{array}{c}
\delta \Delta \underline{\mathbf{u}} \\
\delta \Delta \alpha
\end{array}\right\}^{T}\left(\left\langle\left[\mathbf{B}^{\mathbf{x}+\mathbf{u}}, \mathbf{E}\right]^{T} \boldsymbol{\sigma}\right\rangle+\left(\mathbf{K}_{\sigma}+\left\langle\left[\mathbf{B}^{\mathbf{x}+\mathbf{u}}, \mathbf{E}\right]^{T} \mathbf{C}\left[\mathbf{B}^{\mathbf{x}+\mathbf{u}}, \mathbf{E}\right]\right\rangle\right)\left\{\begin{array}{c}
\Delta \underline{\mathbf{u}} \\
\Delta \alpha
\end{array}\right\}\right)
$$

in which

$\left\langle\left[\mathbf{B}^{\mathbf{x}+\mathbf{u}}, \mathbf{E}\right]^{T} \boldsymbol{\sigma}\right\rangle$ is the equivalent nodal force of the element stress,

$\mathbf{K}_{\sigma}$ is the symmetric initial stress matrix, i.e. $\mathbf{K}_{\sigma}\left\{\begin{array}{c}\Delta \underline{\mathbf{u}} \\ \Delta \alpha\end{array}\right\}=\left\langle\left[\mathbf{B}^{\Delta \mathbf{u}}, \mathbf{0}\right]^{T} \boldsymbol{\sigma}\right\rangle$,

$\left\langle\left[\mathbf{B}^{\mathbf{x}+\mathbf{u}}, \mathbf{E}\right]^{T} \mathbf{C}\left[\mathbf{B}^{\mathbf{x}+\mathbf{u}}, \mathbf{E}\right]\right\rangle$ is the tangential stiffness matrix.

With four sampling points in the URI, the tangential stiffness matrix can be expressed as:

$$
\left\langle\left[\mathbf{B}^{\mathbf{x}+\mathbf{u}}, \mathbf{E}\right]^{T} \mathbf{C}\left[\mathbf{B}^{\mathbf{x}+\mathbf{u}}, \mathbf{E}\right]\right\rangle_{l}=\overline{\mathbf{B}}^{T} \overline{\mathbf{C}} \overline{\mathbf{B}}
$$

where

$$
\overline{\mathbf{B}}=\left[\begin{array}{c}
\left(\left[\mathbf{B}^{\mathbf{x}+\mathbf{u}}, \mathrm{E}\right]\right)_{I} \\
\left(\left[\mathbf{B}^{\mathbf{x}+\mathbf{u}}, \mathrm{E}\right]\right)_{I I} \\
\left(\left[\mathbf{B}^{\mathbf{x}+\mathbf{u}}, \mathrm{E}\right]\right)_{I I I} \\
\left(\left[\mathbf{B}^{\mathbf{x}+\mathbf{u}}, \mathrm{E}\right]\right)_{I V}
\end{array}\right], \overline{\mathbf{C}}=2 \pi\left[\begin{array}{cccc}
(r J \mathbf{C})_{I} & \mathbf{0} & \mathbf{0} & \mathbf{0} \\
\mathbf{0} & (r J \mathbf{C})_{I I} & \mathbf{0} & \mathbf{0} \\
\mathbf{0} & \mathbf{0} & (r J \mathbf{C})_{I I I} & \mathbf{0} \\
\mathbf{0} & \mathbf{0} & \mathbf{0} & (r J \mathbf{C})_{I V}
\end{array}\right]
$$

Suffix " $l$ " denotes that that a lower order $(2 \times 2$ quadrature $)$ integration rule is employed and ()$_{J}$ denotes the value of the braced term at the $J$-th sampling point, see Figure 1.

Same as the Q8 and Q9 URI elements [25], the enhanced Q8 and Q9 URI elements whose displacement-derived strain is enhanced with the volumetric EAS exhibit spurious zero energy mode(s). For a square element with edges parallel to the $r$-and $z$-axes, the modes are:

$$
\left\{\begin{array}{l}
u_{r} \\
u_{z}
\end{array}\right\}=\left\{\begin{array}{c}
p_{\xi} \\
-p_{\eta}
\end{array}\right\}=\left\{\begin{array}{c}
\xi\left(3 \eta^{2}-1\right) \\
-\eta\left(3 \xi^{2}-1\right)
\end{array}\right\},\left\{\begin{array}{l}
u_{r} \\
u_{z}
\end{array}\right\}=\frac{1}{6}\left(3 \xi^{2}-1\right)\left(3 \eta^{2}-1\right)\left\{\begin{array}{l}
c_{1} \\
c_{2}
\end{array}\right\}
$$

in which $p_{\xi}$ and $p_{\eta}$ are self-defined and portrayed in Figure 2; $c$ 's are coefficients. The first mode exists in both elements. However, it rarely causes problem as it is incompatible, i.e., it is self-suppressed when two or more elements are used in a mesh. The last two modes exit only in the Q9 URI elements. However, they are compatible and can plague practical analyses. In the next section, a stabilization scheme is developed for the element. 


\section{LOWER AND HIGHER ORDER HYBRID-STRAIN MODES}

In hybrid-stabilization [9,23,26-28], a hybrid-stress or hybrid-strain field which is independent of the displacement-derived strain field is assumed. The assumed "hybrid" field is partitioned into a lower order " $l$ " and higher order " $h$ " sub-fields. For nonlinear material problems, it is more convenient to use a hybrid-strain field which, for axisymmetric problems, can be expressed as:

$$
\mathbf{e}=\left\{e_{\theta \theta}, e_{r r}, e_{z z}, 2 e \varepsilon_{r z}\right\}^{T}=\mathbf{P}_{l} \boldsymbol{\beta}_{l}+\mathbf{P}_{h} \boldsymbol{\beta}_{h}
$$

where Ps are the shape function matrices and $\beta$ s are the vectors of coefficients. Moreover, one picks

$$
\mathbf{P}_{l}=\left[\mathbf{I}_{4}, \xi \mathbf{I}_{4}, \eta \mathbf{I}_{4}, \xi \eta \mathbf{I}_{4}\right]
$$

Without affecting the basis, the shape matrix can be changed to

$$
\mathbf{P}_{l}=\frac{\sqrt{3}}{4}\left[\left(\frac{1}{\sqrt{3}}-\xi\right)\left(\frac{1}{\sqrt{3}}-\eta\right) \mathbf{I}_{4},\left(\frac{1}{\sqrt{3}}+\xi\right)\left(\frac{1}{\sqrt{3}}-\eta\right) \mathbf{I}_{4},\left(\frac{1}{\sqrt{3}}+\xi\right)\left(\frac{1}{\sqrt{3}}+\eta\right) \mathbf{I}_{4},\left(\frac{1}{\sqrt{3}}-\xi\right)\left(\frac{1}{\sqrt{3}}+\eta\right) \mathbf{I}_{4}\right]
$$

It can be noted that $(1 / \sqrt{ } 3 \pm \xi)(1 / \sqrt{ } 3 \pm \eta)$ equals either 1 or 0 at the $2 \times 2$ quadrature points. The property would facilitate the subsequent proof for showing that the lower order modes will lead to the URI element. In this light, the higher order modes can be chosen to suppress the compatible spurious zero energy modes of the URI element. The strain associated with the displacement in (14) can be derived as:

$$
\left\{\begin{array}{c}
\varepsilon_{\theta \theta} \\
\varepsilon_{\xi \xi} \\
\varepsilon_{\eta \eta} \\
2 \varepsilon_{\xi \eta}
\end{array}\right\}=\left[\begin{array}{cc}
\left(3 \xi^{2}-1\right)\left(3 \eta^{2}-1\right) / 6 & 0 \\
p_{\xi} & 0 \\
0 & p_{\eta} \\
p_{\eta} & p_{\xi}
\end{array}\right]\left\{\begin{array}{l}
c_{1} \\
c_{2}
\end{array}\right\}+\text { second order terms of } c_{1} \text { and } c_{2} \text {. }
$$

To suppress the two spurious modes, the higher order hybrid-strain shape function matrix can be chosen to be:

$$
\boldsymbol{P}_{h}=\frac{1}{r J}\left(\mathbf{T}^{T} \mathbf{C}_{o}\right)^{-1}\left[\begin{array}{cc}
0 & 0 \\
p_{\xi} & 0 \\
0 & p_{\eta} \\
0 & 0
\end{array}\right]
$$

where $\mathbf{C}_{o}$ is the tangential elasticity matrix at zero strain. 


\section{WEAK FORMS FOR THE STABILIZATION}

Let $(\mathbf{u}, \alpha, \mathbf{e})$ and $(\mathbf{u}+\Delta \mathbf{u}, \alpha+\Delta \alpha, \mathbf{e}+\Delta \mathbf{e})$ be the acquired and refined approximate solution to the nonlinear equilibrium problem. The following weak forms can be used [23,29]:

$$
\begin{gathered}
\left\langle(\boldsymbol{\varepsilon}+\Delta \boldsymbol{\varepsilon}-\mathbf{e}-\Delta \mathbf{e})^{T} \mathbf{C} \delta \Delta \mathbf{e}\right\rangle=0 \\
\sum_{e} \delta U^{e}=\delta W
\end{gathered}
$$

The first equation enforces the equality of the enhanced strain $\varepsilon+\Delta \varepsilon$ and the hybrid-strain $\mathbf{e}+\Delta \mathbf{e}$. Moreover,

$$
\delta U^{e}=\left\langle(\boldsymbol{\sigma}+\mathbf{C} \Delta \mathbf{e})^{T} \delta \Delta \boldsymbol{\varepsilon}\right\rangle
$$

in which $\mathbf{C} \Delta \mathbf{e}$ replaces $\mathbf{C} \Delta \boldsymbol{\varepsilon}$ in (7) for the enhanced URI element. Similarly,

$$
\boldsymbol{\sigma}=\frac{\partial A(\mathbf{e})}{\partial \mathbf{e}}, \mathbf{C}=\frac{\partial}{\partial \mathbf{e}}\left(\frac{\partial A(\mathbf{e})}{\partial \mathbf{e}}\right)^{T}
$$

Further from (13),

$$
\Delta \mathbf{e}=\left[\mathbf{P}_{l}, \mathbf{P}_{h}\right]\left\{\begin{array}{c}
\Delta \boldsymbol{\beta}_{l} \\
\Delta \boldsymbol{\beta}_{h}
\end{array}\right\}, \delta \Delta \mathbf{e}=\left[\mathbf{P}_{l}, \mathbf{P}_{h}\right]\left\{\begin{array}{l}
\delta \Delta \boldsymbol{\beta}_{l} \\
\delta \Delta \boldsymbol{\beta}_{h}
\end{array}\right\}
$$

After invoking (9) and (20), linearization of (16) and (18) with respect to the incremental terms leads to

$$
\begin{gathered}
\left\langle\left[\begin{array}{c}
\mathbf{P}_{l}^{T} \\
\mathbf{P}_{h}^{T}
\end{array}\right] \mathbf{C}\left(\boldsymbol{\varepsilon}-\mathbf{e}+\left[\mathbf{B}^{\mathbf{x}+\mathbf{u}}, \mathbf{E}\right]\left\{\begin{array}{c}
\Delta \underline{\mathbf{u}} \\
\Delta \alpha
\end{array}\right\}-\left[\mathbf{P}_{l}, \mathbf{P}_{h}\right]\left\{\begin{array}{c}
\Delta \boldsymbol{\beta}_{l} \\
\Delta \boldsymbol{\beta}_{h}
\end{array}\right\}\right)\right\rangle=\mathbf{0}, \\
\delta U^{e}=\left\{\begin{array}{c}
\delta \Delta \underline{\mathbf{u}} \\
\delta \Delta \alpha
\end{array}\right\}^{T}\left\langle\left[\mathbf{B}^{\mathbf{x}+\mathbf{u}+\Delta \mathbf{u}}, \mathbf{E}\right]^{T} \boldsymbol{\sigma}+\left[\mathbf{B}^{\mathbf{x}+\mathbf{u}}, \mathbf{E}\right]^{T} \mathbf{C}\left[\mathbf{P}_{l}, \mathbf{P}_{h}\right]\left\{\begin{array}{c}
\Delta \boldsymbol{\beta}_{l} \\
\Delta \boldsymbol{\beta}_{h}
\end{array}\right\}\right\rangle
\end{gathered}
$$

While \langle\rangle$_{l}$ denotes the domain integration by the lower (second) order quadrature, let \langle\rangle$_{h}$ denote the domain integration by the higher (third) order quadrature. Using the standard orders of integration [10] and noting that $\mathbf{P}_{h}$ vanishes at all second order quadrature points, (21) can be expanded as:

$$
\mathbf{Z}+\mathbf{G}\left\{\begin{array}{c}
\Delta \underline{\mathbf{u}} \\
\Delta \alpha
\end{array}\right\}-\mathbf{H}\left\{\begin{array}{c}
\Delta \boldsymbol{\beta}_{l} \\
\Delta \boldsymbol{\beta}_{h}
\end{array}\right\}=\mathbf{0}, \delta U^{e}=\left\{\begin{array}{c}
\delta \Delta \underline{\mathbf{u}} \\
\delta \Delta \alpha
\end{array}\right\}^{T}\left(\left\langle\left[\mathbf{B}^{\mathbf{x}+\mathbf{u}+\Delta \mathbf{u}}, \mathbf{E}\right]^{T} \boldsymbol{\sigma}\right\rangle+\mathbf{G}^{T}\left\{\begin{array}{c}
\Delta \boldsymbol{\beta}_{l} \\
\Delta \boldsymbol{\beta}_{h}
\end{array}\right\}\right)
$$

in which

$$
\mathbf{H}=\left[\begin{array}{cc}
\left\langle\mathbf{P}_{l}^{T} \mathbf{C} \mathbf{P}_{l}\right\rangle_{l} & \left\langle\mathbf{P}_{l}^{T} \mathbf{C} \mathbf{P}_{h}\right\rangle_{l} \\
\left\langle\mathbf{P}_{h}^{T} \mathbf{C} \mathbf{P}_{l}\right\rangle_{l} & \left\langle\mathbf{P}_{h}^{T} \mathbf{C P}_{h}\right\rangle_{h}
\end{array}\right]=\left[\begin{array}{cc}
\mathbf{H}_{l} & \mathbf{0} \\
\mathbf{0} & \mathbf{H}_{h}
\end{array}\right], \mathbf{G}=\left[\begin{array}{c}
\mathbf{G}_{l} \\
\mathbf{G}_{h}
\end{array}\right]=\left[\begin{array}{l}
\left\langle\mathbf{P}_{l}^{T} \mathbf{C}\left[\mathbf{B}^{\mathbf{x}+\mathbf{u}}, \mathbf{E}\right]\right\rangle_{l} \\
\left\langle\mathbf{P}_{h}^{T} \mathbf{C}\left[\mathbf{B}^{\mathbf{x}+\mathbf{u}}, \mathbf{E}\right]\right\rangle_{h}
\end{array}\right],
$$




$$
\mathbf{Z}=\left[\begin{array}{c}
\mathbf{Z}_{l} \\
\mathbf{Z}_{h}
\end{array}\right]=\left[\begin{array}{c}
\left\langle\mathbf{P}_{l}^{T} \mathbf{C}(\boldsymbol{\varepsilon}-\mathbf{e})\right\rangle_{l} \\
\left\langle\mathbf{P}_{h}^{T} \mathbf{C}(\boldsymbol{\varepsilon}-\mathbf{e})\right\rangle_{h}
\end{array}\right]
$$

The unspecified integration order will be later discussed. From the first expression in (22),

$$
\left\{\begin{array}{c}
\Delta \boldsymbol{\beta}_{l} \\
\Delta \boldsymbol{\beta}_{h}
\end{array}\right\}=\left[\begin{array}{c}
\mathbf{H}_{l}^{-1} \mathbf{Z}_{l} \\
\mathbf{H}_{h}^{-1} \mathbf{Z}_{h}
\end{array}\right]+\left[\begin{array}{c}
\mathbf{H}_{l}^{-1} \mathbf{G}_{l} \\
\mathbf{H}_{h}^{-1} \mathbf{G}_{h}
\end{array}\right]\left\{\begin{array}{c}
\Delta \underline{\mathbf{u}} \\
\Delta \alpha
\end{array}\right\}
$$

with which the second expression in (22) becomes:

$$
\delta U^{e}=\left\{\begin{array}{c}
\delta \Delta \underline{\mathbf{u}} \\
\delta \Delta \alpha
\end{array}\right\}^{T}\left(\left\langle\left[\mathbf{B}^{\mathbf{x}+\mathbf{u}+\Delta \mathbf{u}}, \mathbf{E}\right]^{T} \boldsymbol{\sigma}\right\rangle+\mathbf{G}_{l}^{T} \mathbf{H}_{l}^{-1} \mathbf{Z}_{l}+\mathbf{G}_{h}^{T} \mathbf{H}_{h}^{-1} \mathbf{Z}_{h}+\left(\mathbf{G}_{l}^{T} \mathbf{H}_{l}^{-1} \mathbf{G}_{l}+\mathbf{G}_{h}^{T} \mathbf{H}_{h}^{-1} \mathbf{G}_{h}\right)\left\{\begin{array}{c}
\Delta \underline{\mathbf{u}} \\
\Delta \alpha
\end{array}\right\}\right)
$$

\section{FURTHER MANIPULATIONS AND SIMPLICATIONS}

With the chosen lower order hybrid-strain shape function matrix $\mathbf{P}_{l}$ in (13), it is trivial to show that some of the terms defined below (22) and appeared in (23) and (24) can be expressed as:

$$
\mathbf{G}_{l}=\overline{\mathbf{C}} \overline{\mathbf{B}}, \mathbf{H}_{l}=\overline{\mathbf{C}}, \mathbf{Z}_{l}=2 \pi\left[\begin{array}{c}
(r J \mathbf{C}(\boldsymbol{\varepsilon}-\mathbf{e}))_{I} \\
(r J \mathbf{C}(\boldsymbol{\varepsilon}-\mathbf{e}))_{I I} \\
(r J \mathbf{C}(\boldsymbol{\varepsilon}-\mathbf{e}))_{I I I} \\
(r J \mathbf{C}(\boldsymbol{\varepsilon}-\mathbf{e}))_{I V}
\end{array}\right], \mathbf{G}_{l}^{T} \mathbf{H}_{l}^{-1} \mathbf{Z}_{l}=\left\langle\left[\mathbf{B}^{\mathbf{x}+\mathbf{u}}, \mathbf{E}\right]^{T} \mathbf{C}(\boldsymbol{\varepsilon}-\mathbf{e})\right\rangle_{l}
$$

where $\overline{\mathbf{B}}$ and $\overline{\mathbf{C}}$ have been defined in (11). Furthermore, if $\mathbf{Z}_{h}$ and the term involving the stress $\boldsymbol{\sigma}$ in (24) are evaluated by the $2 \times 2$ quadrature, the former vanishes and from (25),

$$
\left\langle\left[\mathbf{B}^{\mathbf{x}+\mathbf{u}+\Delta \mathbf{u}}, \mathbf{E}\right]^{T} \boldsymbol{\sigma}\right\rangle_{l}+\mathbf{G}_{l}^{T} \mathbf{H}_{l}^{-1} \mathbf{Z}_{l}=\underline{\left\langle\left[\mathbf{B}^{\mathbf{x}+\mathbf{u}}, \mathbf{E}\right]^{T}(\boldsymbol{\sigma}+\mathbf{C}(\boldsymbol{\varepsilon}-\mathbf{e}))\right\rangle_{l}}+\mathbf{K}_{\sigma}\left\{\begin{array}{c}
\Delta \underline{\mathbf{u}} \\
\Delta \alpha
\end{array}\right\}
$$

where the underlined term represents the lumped nodal equivalent effect of the stress and the mismatch of the enhanced and hybrid strains; $\mathbf{K}_{\sigma}$ is the initial stress defined under (10). Thus, (24) is simplified to

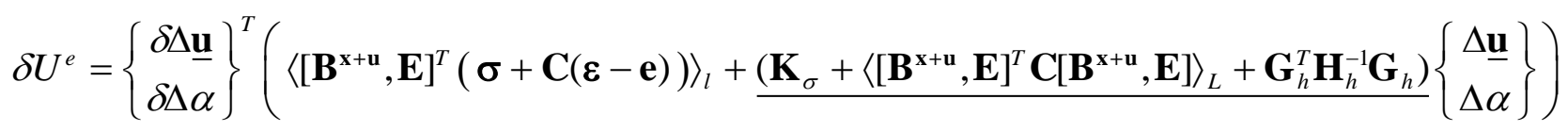

in which the underlined term is the generalized tangential element stiffness matrix. Compared with the generalized tangential element stiffness matrix of the uniformly reduced integrated element, the only extra term is $\mathbf{G}_{h}^{T} \mathbf{H}_{h}^{-1} \mathbf{G}_{h}$ which plays the role of stabilizing the element. Noting that the number of higher order hybrid strain modes is 2 whereas total number of nodal d.o.f.s and EAS modes is 19, the 
accuracy of element should be insensitive to whether $\mathbf{H}_{h}$ and $\mathbf{G}_{h}$ are legitimately evaluated. For higher computational efficiency, the tangential material stiffness matrices in $\mathbf{H}_{h}$ and $\mathbf{G}_{h}$ are approximated by its strain-free counterpart, i.e. $\mathbf{C} \simeq \mathbf{C}_{o}$. In this light, $\mathbf{H}_{h}$ and $\mathbf{G}_{h}$ can then be simplified to:

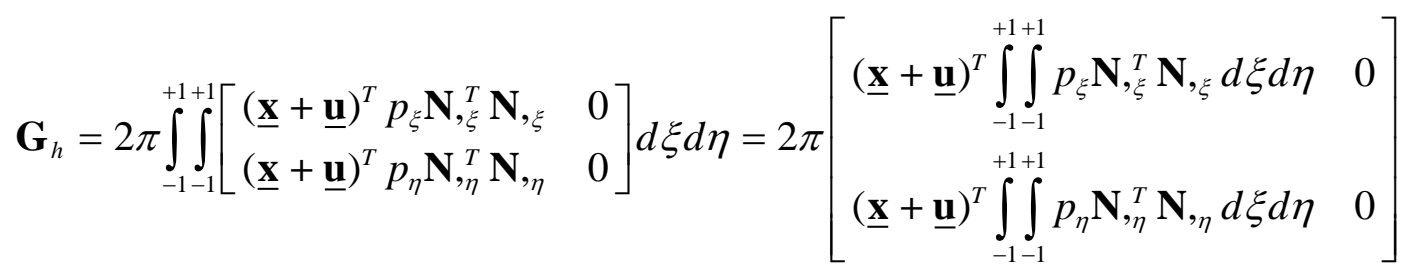

and

$$
\boldsymbol{H}_{h}=2 \pi \int_{-1}^{+1} \int_{-1}^{+1} r J \boldsymbol{P}_{h}^{T} \mathbf{C}_{o} \boldsymbol{P}_{h} d \xi d \eta=2 \pi \int_{-1}^{+1} \int_{-1}^{+1} \frac{1}{r J}\left[\begin{array}{ll}
p_{\xi} p_{\xi} s_{22} & p_{\xi} p_{\eta} s_{23} \\
p_{\xi} p_{\eta} s_{23} & p_{\eta} p_{\eta} s_{33}
\end{array}\right] d \xi d \eta
$$

where $\left[s_{i j}\right]=\mathbf{T}^{-1} \mathbf{C}_{o}^{-1} \mathbf{T}^{-T}$. The matrices arising from two integrals at the right hand side of (28) are independent of the element geometry and displacement constant. They can be derived explicitly. On the other hand, by further approximating $\left[s_{i j}\right]$ and $r J$ with their counterparts at the element centre,

$$
\boldsymbol{H}_{h}=\left.\frac{16 \pi}{5}\left(\frac{1}{r J}\left[\begin{array}{cc}
s_{22} & 0 \\
0 & s_{33}
\end{array}\right]\right)\right|_{\xi=\eta=0}
$$

To summarize, the stabilization scheme do not require the use of numerical integration.

\section{NUMERICAL ILLUSTRATIONS}

In this section, the presented elements are examined by a number of linear and nonlinear problems. In the nonlinear solution procedure, the full Newton-Raphson iteration method is employed. The solution is deemed to be converged if the $0.5 \%$ force tolerance and $1 \%$ displacement tolerance are simultaneously satisfied. The criteria are identical to the default ones used by ABAQUS [24]. The following axisymmetric elements would be included in our comparison:

Q8 - the URI Q8 element;

Q8H - the u-p formulated URI Q8 element with three pressure modes;

EQ8 - the enhanced (with a volumetric EAS mode) URI Q8 element, see Section 3;

EQ9 -the enhanced (with a volumetric EAS mode) and stabilized URI Q9 element, see Section 5.

In ABAQUS, Q8 and Q8H are designated as CAX8R and CAX8RH, respectively. Their predictions are computed by ABAQUS. In the examples, the default units for length and force are $\mathrm{cm}$ and $\mathrm{kgf}$, 
respectively. Thus, the unit for stress, pressure and material modulus is $\mathrm{kgf} / \mathrm{cm}^{2}$. Neo-Hookean materials are assumed in the nonlinear examples and the pertinent strain energy potential is:

$$
W=C\left(\bar{I}_{1}-3\right)+\frac{1}{2} K(J-1)^{2} \quad \bar{I}_{1}=I_{1} I_{3}^{-1 / 3}, \quad J=I_{3}^{1 / 2}
$$

in which $I_{1}$ and $I_{3}$ are respectively the first and third invariants of Green-Lagrangian strain; the material constant $C$ is taken to be $50 \mathrm{kgf} / \mathrm{cm}^{2}$ and $K / C$ is taken to be either 10 or 10000 . Under infinitesimal displacement, $2 C$ and $K$ are the same as respectively the shear and bulk modului whereas $K / C=10$ and 10000 correspond to Poisson's ratios 0.406 and 0.4999 , respectively.

\subsection{Patch Test}

The five-element mesh of MacNeal and Harder [30] is employed for patch test as shown in Figure 3. The top and bottom boundaries of the mesh are restrained from moving along the $\mathrm{z}$-direction. The inner and outer radii are prescribed with the same pressure. The test is geometric and material linear problem. The exact constant stress solution is reproduced by all element models.

\subsection{Plane Strain Annulus subjected to Internal Pressure}

Figure 4 shows a plane strain annulus with inner radius 5 , outer radius 8 and unit thickness. A $5 \times 1$ mesh is employed. The problem is commonly used to examine the element performance with respect to volumetric locking. The maximum pressure is taken to be 30 . All the element models yield graphically indistinguishable radial displacements at the inner and outer radii for both $K / C$ ratios, see Figure 5. In this problem, there is no sign of dilatational locking in all elements. The deformation reduces as $K / C$ increases.

\subsection{Spherical Membrane subjected to Internal Pressure}

In this example, a spherical membrane of inner radius 10 and thickness 0.1 is subjected to internal pressure with maximum value 1.2 . Owing to symmetry, only half of the membrane is modelled as shown in Figure 6. Figure 7 plots the radial deflection at $z=0$. An $1 \times 40$ mesh is employed. Same as the last example, all element models produce the graphically indistinguishable predictions. Moreover, with a pressure increment of 0.12 and at the $K / C=10$, all element models fails to converged when the pressure exceeds 0.96 . The plotted radial deflections at $z=0$ have also been compared with the 
longitudinal deflections at $r=0$. The two deflections are also graphically indistinguishable. Again, there is no sign of dilatational locking and the deformation reduces as $K / C$ increases.

\subsection{Simply-Supported Circular Plate}

Figure 8 shows a $\phi 20 \times 1$ simply-supported circular plate subjected to a maximum transverse pressure of 2 unit. An $1 \times 8$ mesh is employed. In the problem, the lower right-hand node is restrained from vertical movement. As usual, the nodes on the z-axis are restrained from radial movement. The central downward deflection immediately under the load is extracted and plotted in Figure 9. All elements yield graphically indistinguishable predictions when $K / C=10$. However, with $K / C=10000$, Q8 fails to converge in the first load step when the pressure equals 0.2 unit. For the convergent elements, their predictions are again graphically indistinguishable.

\subsection{Non-leaky Cavity Problem}

The problem is modified from the well-known non-leaky cavity flow problem [4,9] which is a highly restrained and, thus, a very demanding problem for nearly incompressibility material analysis. The $1 \times 1 \mathrm{~cm}$ computational domain in Figure 12 is equally divided into $15 \times 15$ elements. With the two end nodes excluded, the radial displacements of the nodes at $z=1$ are prescribed to be 0.01 . The remaining boundary displacement d.o.f.s are completely restrained. The predicted pressures along the upper $2 \times 2$ Gaussian of the middle element row are extracted. For $K / C=10$, the pressures are shown in Figure 10. The predictions of Q8 and EQ9 are smooth graphically indistinguishable. The predictions of Q8H and EQ8 are indistinguishable. However, they oscillate slightly at the two ends. For $K / C=10$ 000, the pressures are shown in Figure 11. Q8 fails to converge and, thus, its prediction is absent from the figure. Instead of oscillating slightly in Figure 10, QH8 and EQ8 exhibit strong pressure oscillations with the peak-to-peak amplitude approximately equal to 200 . On the other hand, the pressure predicted by EQ9 is smooth and varies between 15 and 45 . 


\section{CLOSURE}

In this paper, an 8-node quadrilateral (Q8) and a 9-node quadrilateral (Q9) axisymmetric elements for large deformation analysis of hyperelastic problems are devised. Both elements are supplemented with an enhanced volumetric strain mode. While both elements are integrated by the $2 \times 2$ quadrature, a stabilization matrix is developed for the latter element to suppress two compatible zero energy mechanisms. In view of constraint counting [10], the enhanced Q8 and Q9 elements are analogous to the hybrid Q8 and Q9 elements with three assumed pressure modes in the u-p formulation. However, the use of enhanced strain mode is more straight forward than the use of pressure modes in both formulation and computer implementation. With an extra interior nodes, the enhanced Q9 element is more robust for nearly incompressible analysis. When the problem domain is not highly restrained, the proposed elements and Abaqus's hybrid URI Q8 (with three assumed pressure modes) yield close predictions. However, when the problem domains are highly constrained and the material is nearly incompressible, pressure predictions of the enhanced Q8 and Abaqus's hybrid URI Q8 elements are oscillatory whereas the predictions of the enhanced Q9 element remains smooth. It is also noted that the conventional URI Q8 element fails to converge more readily than the other elements with devices for alleviating the volumetric locking.

Acknowledgment - The financial support of the University of Hong Kong in the form of a CRGC Small Project Grant is gratefully acknowledged. 


\section{REFERENCES}

[1] D.J. Naylor, Stresses in nearly incompressible materials by finite elements with application to the calculation of excess pore pressures, Inter.J.Numer.Methods Engrg. 8 443-460 (1974)

[2] Pian THH, Lee SW. Notes on finite elements for nearly incompressible materials. AIAA Journal 14: 824826 (1976)

[3] Malkus DS, Hughes TJR. Mixed finite element method - reduced and selective integration techniques: a unification of concepts. Computational Methods in Applied Mechanics \& Engineering 15: 63-81 (1978)

[4] R.L.Sani, P.M.Gresho, R.L.Lee and D.F.Griffiths. "The cause and cure (?) of the spurious pressure generated by certain fem solutions of the incompressible Navier-Stokes equations: Part 1" International Journal for Numerical Methods in Fluids 1: 17-43 (1981)

[5] R.L.Spilker, "Improved hybrid-stress axisymmetric elements including behaviour for nearly incompressible materails", Inter.J.Numer.Methods Engrg., 17, 483-501 (1981)

[6] R.L.Sani, P.M.Gresho, R.L.Lee and D.F.Griffiths. "The cause and cure (?) of the spurious pressure generated by certain fem solutions of the incompressible Navier-Stokes equations: Part 2" International Journal for Numerical Methods in Fluids 1: 171-204 (1981)

[7] H.S. Yu, A rational displacement interpolation function for axisymmetric finite element analysis of incompressible materials, Finite Elements in Analysis and Design 10 (1991) 205-219

[8] H.S. Yu, G.T. Houlsby, H.J. Burd, Novel isoparametric finite element displacement formulation for axisymmetric analysis of nearly incompressible materials, Inter.J. Numer.Methods Engrg. 36: 2453-2472 (1993)

[9] Sze KY, Fan H, Chow CL. Elimination of spurious kinematic and pressure modes in biquadratic plane element. Inter.J. Numer.Methods Engrg. 38: 3911-3932 (1995).

[10] Zienkiewicz OC, Taylor RL. The Finite Element Method. 5th Edn., Butterworth Heinemann, 2000.

[11] H.S. Yu and M.D. Netherton, Performance of displacement finite elements for modeling incompressible materials, Inter.J.Numer. \& Analytical Methods in Geomechanics 24: 627-653 (2000)

[12] R.L.Taylor, P.J.Beresford, E.L.Wilson, "A non-conforming element for stress analysis", Inter.J. Numer.Methods Engrg. 10: 1211-1219 (1976)

[13] J.C.Simo, M.S.Rifai, "A class of mixed assumed strain methods and the method of incompatible modes", Inter.J.Numer.Methods Engrg., 29, 1595-1638 (1990)

[14] K.Y. Sze and C.L. Chow, An incompatible element for axisymmetric structure and its modification by hybrid Method, Inter.J. Numer.Methods Engrg. 31 385-405 (1991)

[15] W.J. Chen and Y.K. Cheung, The nonconforming element method and refined hybrid element method for axisymmetric solid, Inter.J. Numer.Methods Engrg. 39 2509-2529 (1996)

[16] E.P. Kasper and R.L. Taylor, Mixed-enhanced formulation for geometrically linear axisymmetric problems, Inter.J. Numer.Methods Engrg. 53 2061-2086 (2002)

[17] R.L.Spilker, T.H.H.Pian, "A study of axisymmetric solid of revolution elements based on the assumedstress hybrid model, Computers \& Structures, 9, 273-279 (1978)

[18] Z.S. Tian and T.H.H. Pian, Axisymmetric solid elements by a rational hybrid stress method, Computers \& Structures 20: 141-149 (1985)

[19] W.J. Chen and Y.K. Cheung, Axisymmetric solid elements by the generalized hybrid methods, Computers \& Structures 27: 745-752 (1987)

[20] S.L. Weissman and R.L. Taylor, Four-node axisymmetric element based upon the Hellinger-Reissner functional, Computer Methods in Applied Mechanics and Engineering 85: 39-55 (1991)

[21] C.S. Jog and R. Annabattula, The development of hybrid axisymmetric elements based on the HellingerReissner variational principle, Inter.J. Numer.Methods Engrg. 65: 2279-2291 (2006) 
[22] W.E.Bachrach, T.Belytschko, "Axisymmetric elements with high coasrse-mesh accuarcy", Computers \& Structures, 23, 323-331 (1986)

[23] Sze KY, Zheng SJ, Lo SH. A stabilized eighteen-node solid element for hyperelastic analysis of shells. Finite Elements in Analysis \& Design. 40: 319-340 (2004).

[24] Abaqus, ABAQUS/Standard User's Manual. Version 6.3.

[25] Cook RD, Malkus DS, Plesha ME. Concepts and Applications of Finite Element Analysis. $3^{\text {rd }}$ Edn. John Wiley \& Sons, 1989.

[26] Sze KY. A novel approach for devising higher order hybrid elements. Inter.J.Numer. Methods Engrg. 36: 3303-3316 (1993).

[27] Lee SE, Rhiu JJ. A new efficient approach to the formulation of mixed finite element modes for structural analysis. Inter.J.Numer. Methods Engrg. 21: 1629-1641 (1986).

[28] H.C. Park, C. Cho and S.W. Lee, An efficient assumed strain element model with 6 dof per node for geometrically non-linear shells, Inter.J.Numer. Methods Engrg. 38: 4101-4122 (1995).

[29] M.A. Crisfield, Non-linear Finite Element Analysis of Solids and Structures, Volume 2: Advanced Topics, Wiley, 1997.

[30] R.H. RacNeal and R.L. Harder, A proposed standard set of problems to test finite element accuracy, Finite Elements in Analysis and Design 1: 3-20 (1985) 


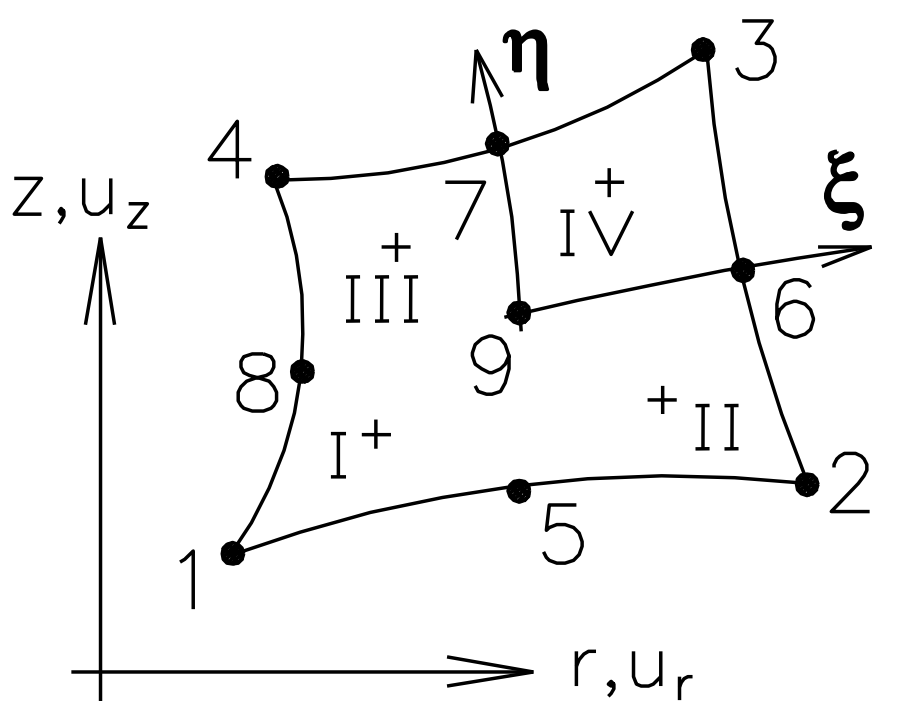

Figure 1. The quadrilateral element on the r-z-plane. Nodes are numbered by Arabic numerals and the $2 \times 2$ quadrature points are numbered by Roman numerals.
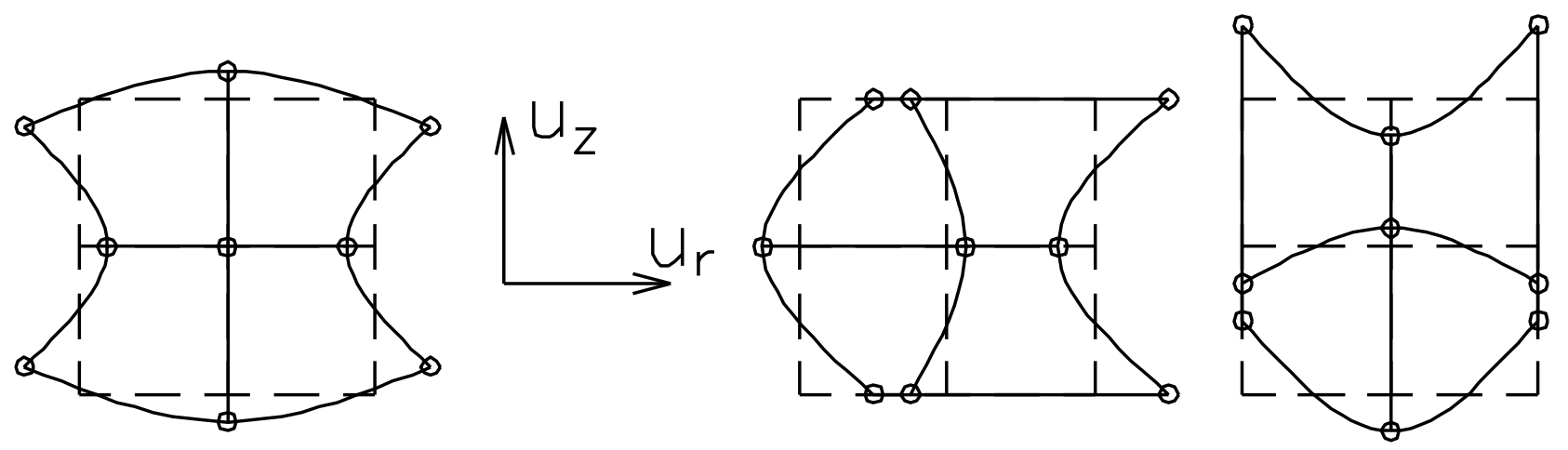

Figure 2. The compatible zero energy mechanism of the Q9 URI element.

$(0.24,0.12) \quad(0.48,0.12)$

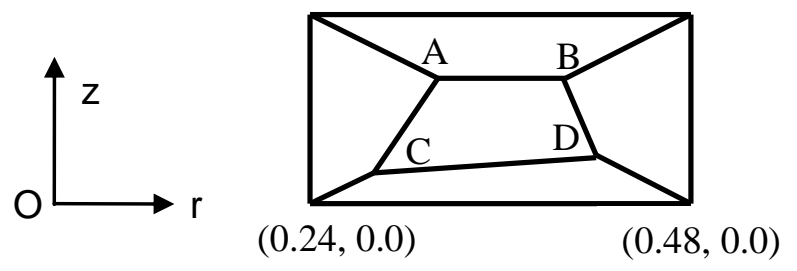

Figure 3. An annular for conducting patch test, the co-ordinates of A, B, C and D are $(0.32,0.08),(0.40,0.08),(0.28,0.02)$ and $(0.42,0.03)$. 


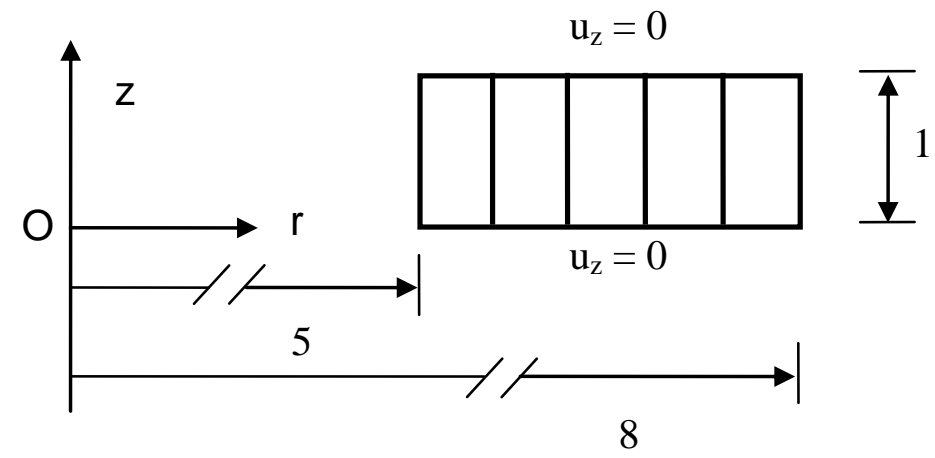

Figure 4. A plane strain annulus subjected to internal pressure and the employed $5 \times 1$ mesh.

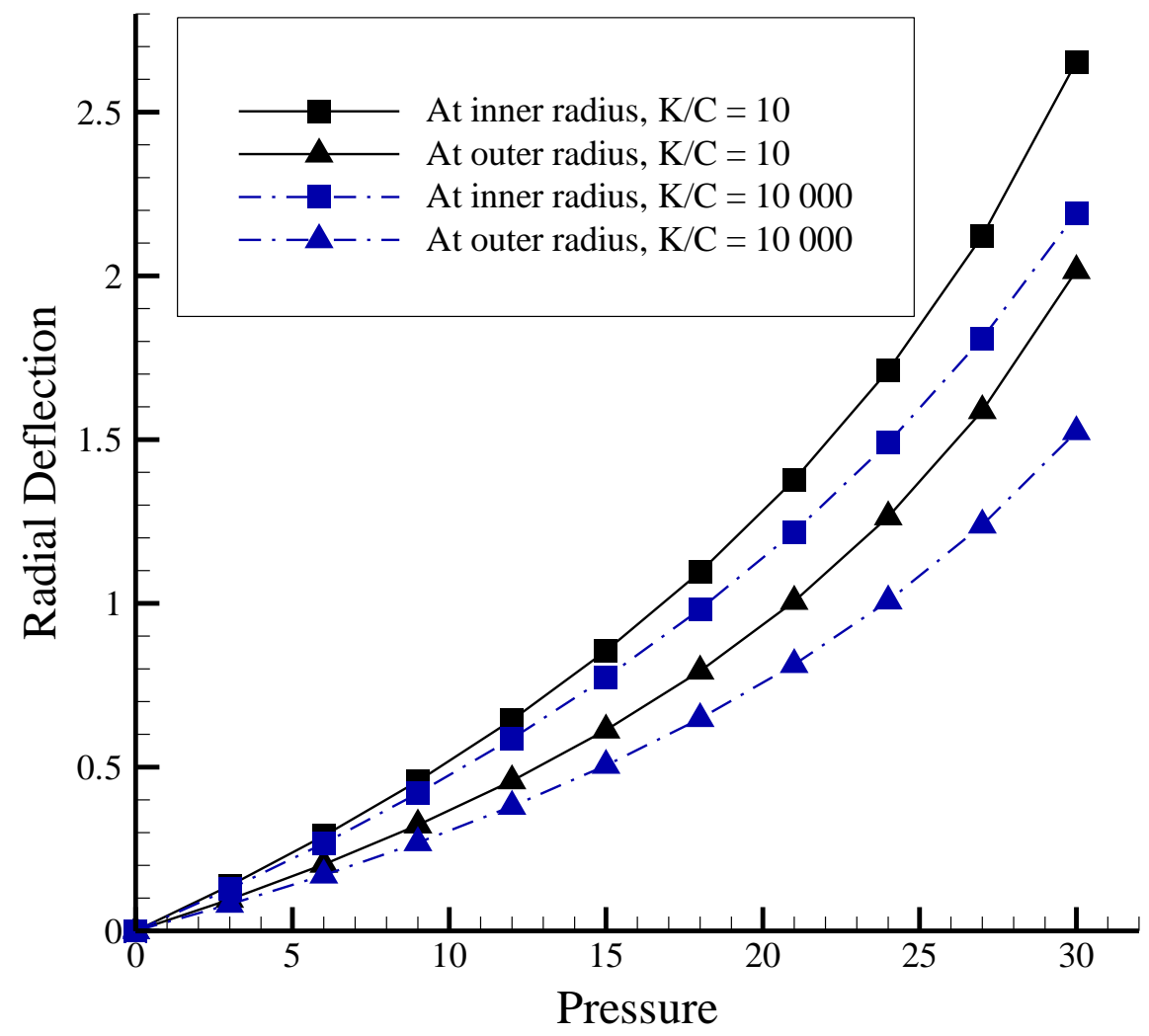

Figure 5. The radial deflections at the inner and outer radii of the annulus problem, see Figure 4. 


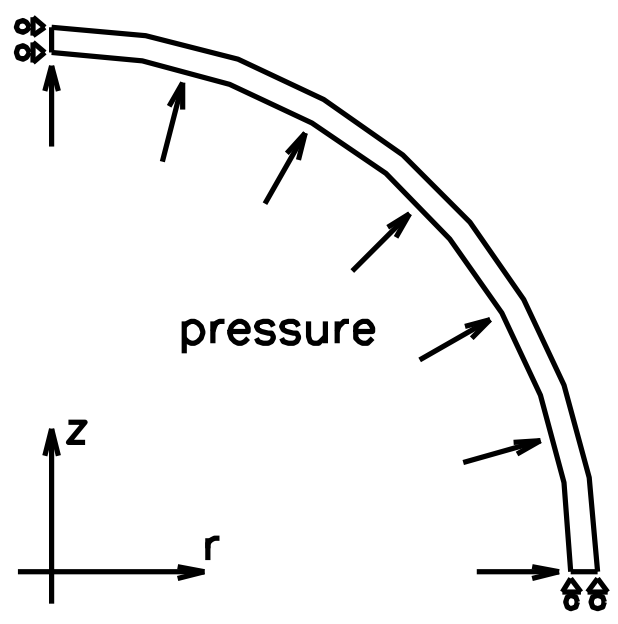

Figure 6. A spherical membrane of inner radius thickness 10 and thickness 0.1 subjected to internal pressure.

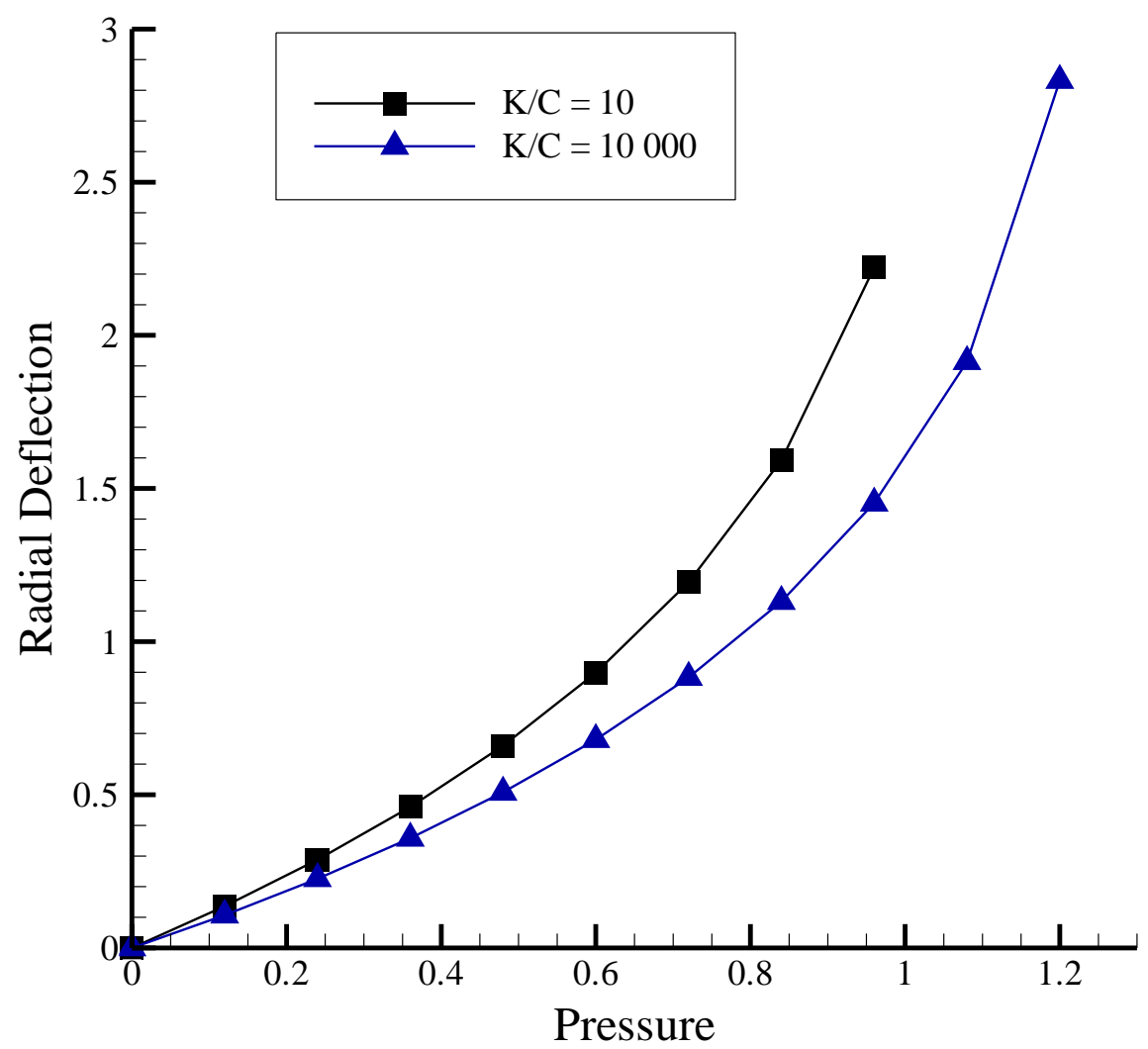

Figure 7. The radial deflection for the spherical membrane problem, see Figure 6. 


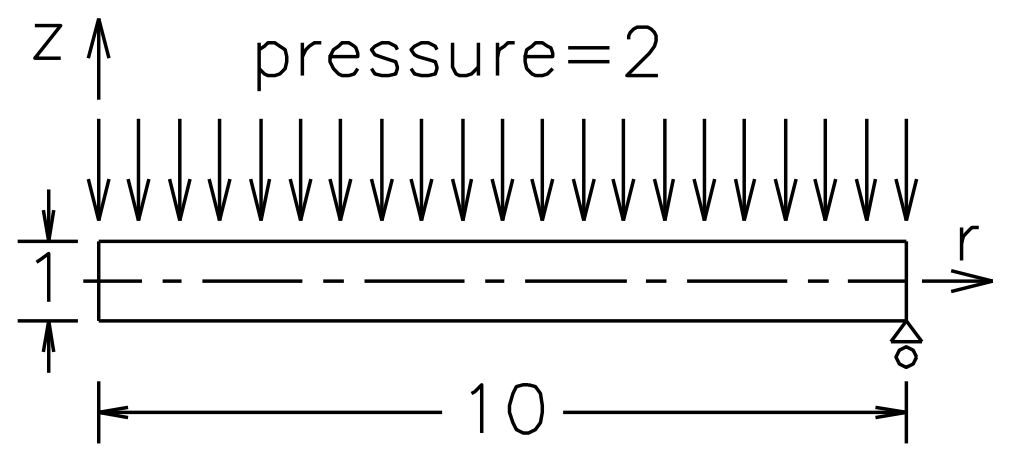

Figure 8. The circular plate problem.

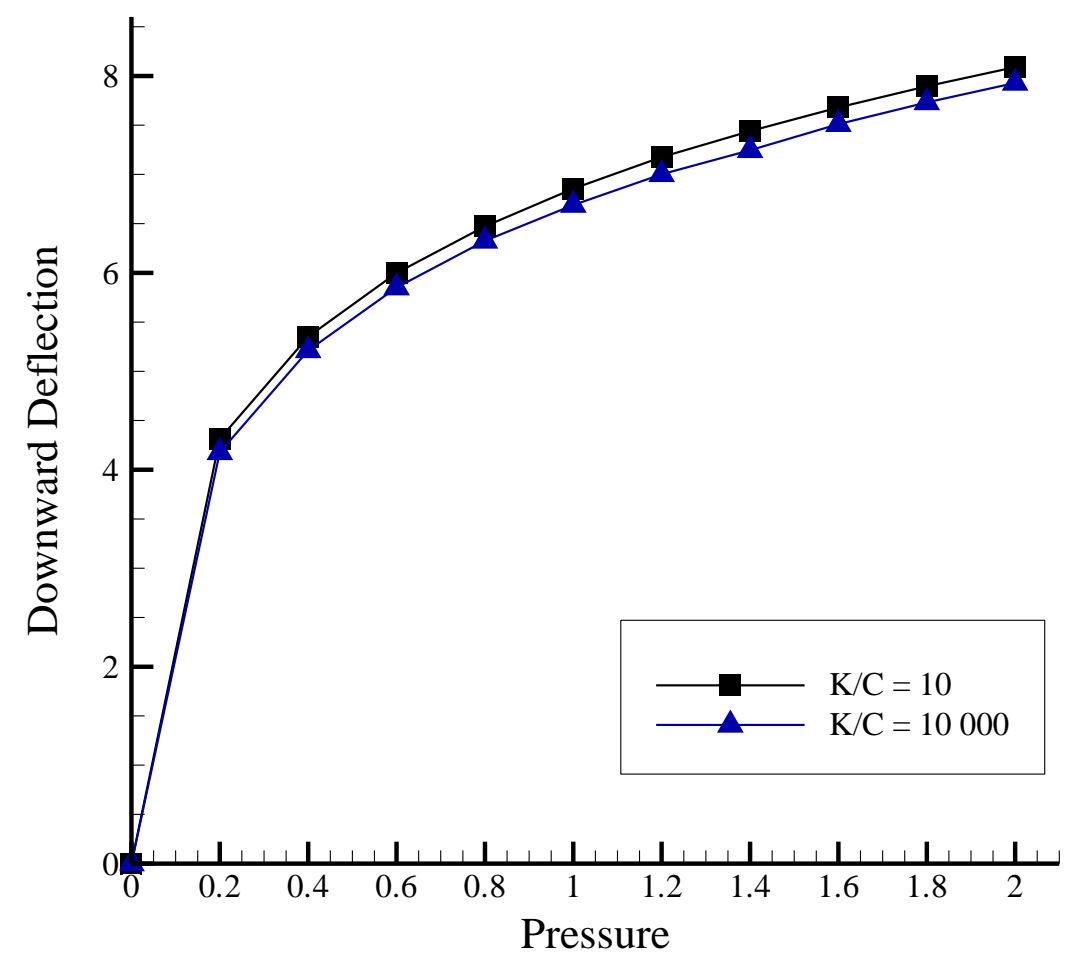

Figure 9. The central downward deflection for the circular plate problem, see Figure 8. 


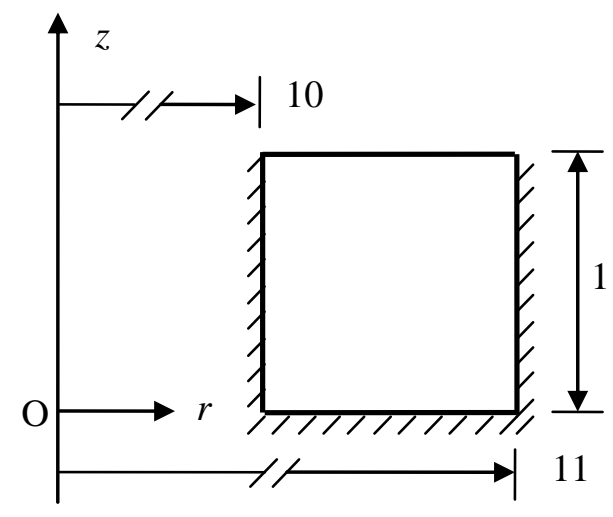

Figure 10. The non-leaky cavity problem. Except the two end nodes which are fully restrained, all nodes over the top face are prescribed with $u_{z}=0$ and $u_{r}=0.01$. A regular $15 \times 15$ mesh is employed.

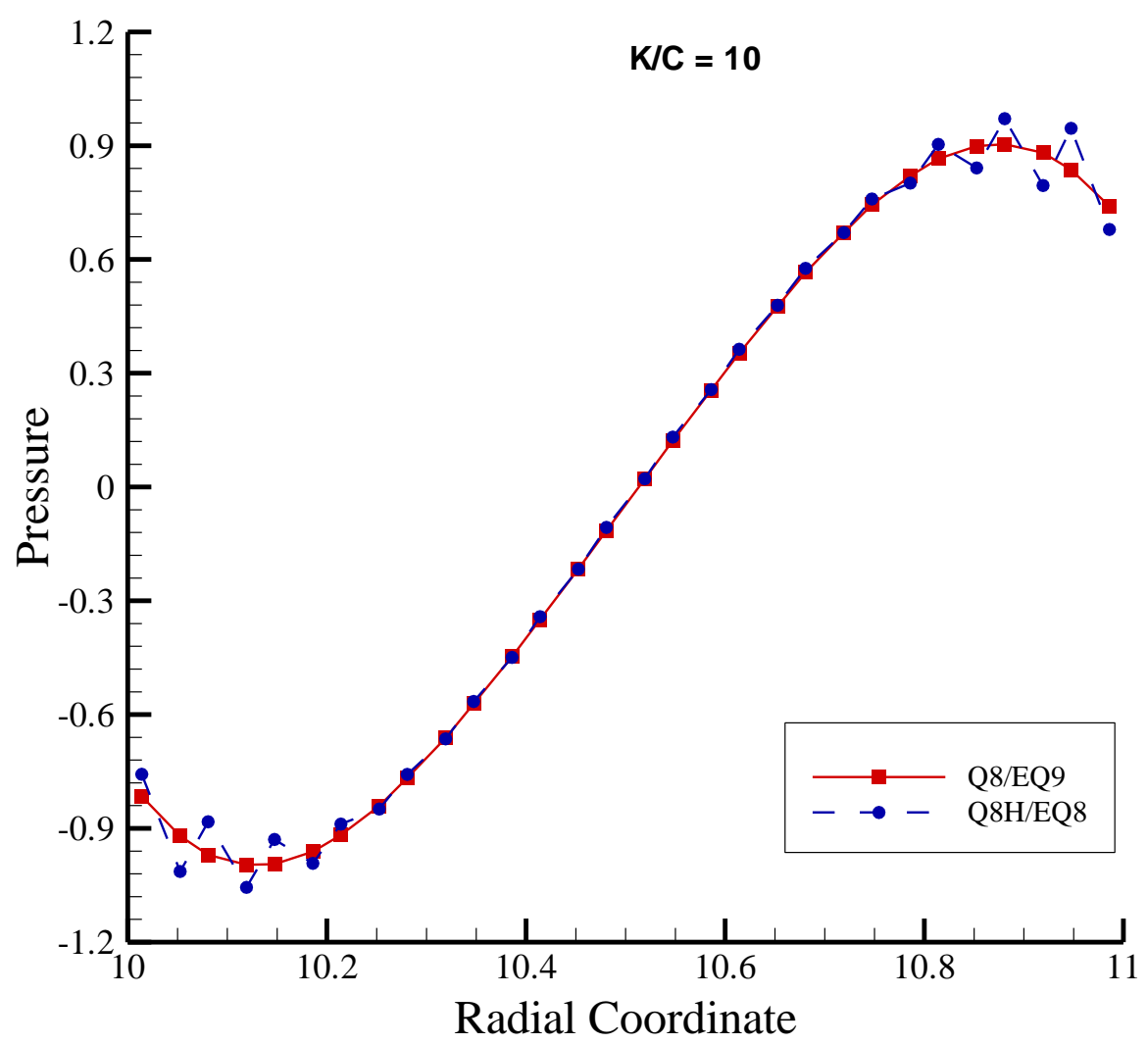

Figure 10. The predicted pressure along the upper $2 \times 2$ Gaussian points of the central element row in the cavity problem when $K / C=10$. 


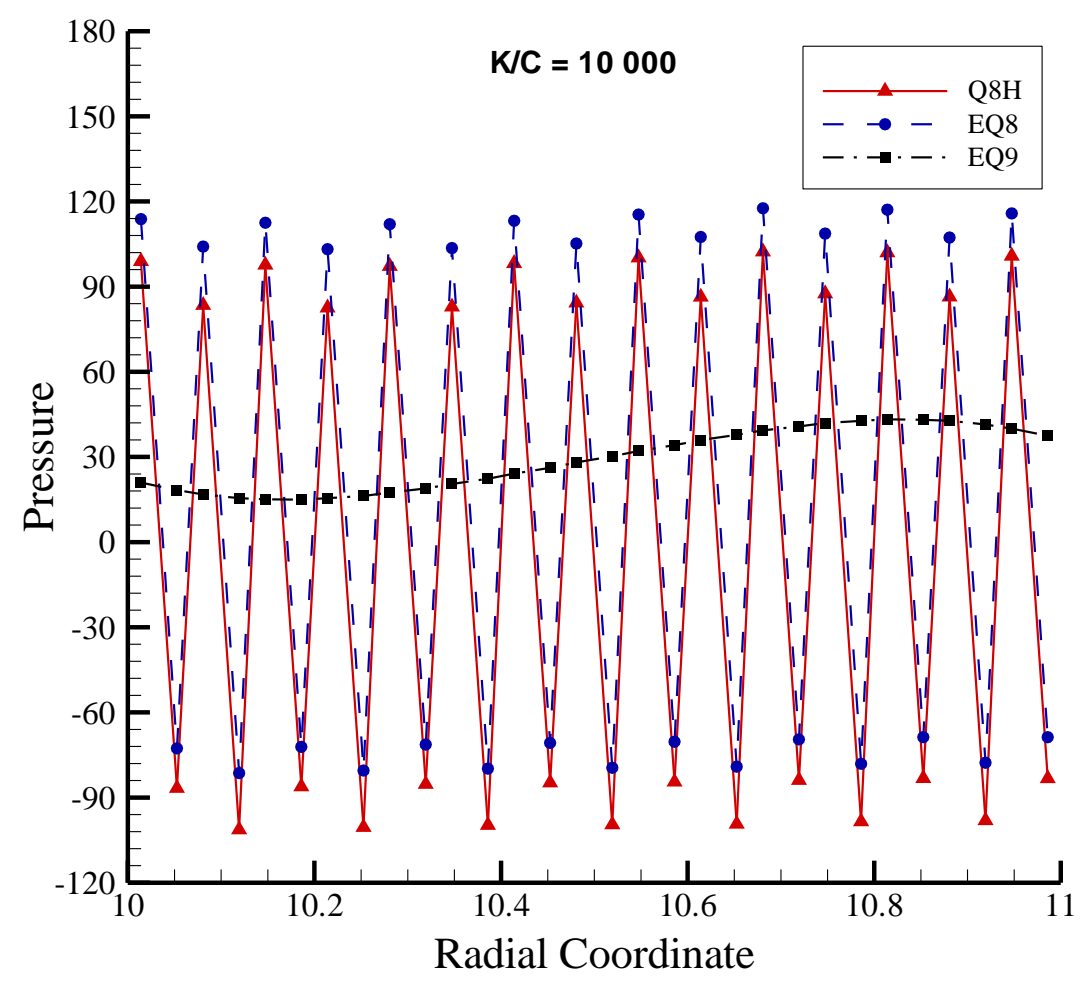

Figure 11 . The predicted pressure along the upper $2 \times 2$ Gaussian points of the central element row in the cavity problem when $K / C=10000$. 